\title{
Convergence and Error of Some Numerical Methods for Solving a Convection-Diffusion Problem
}

\author{
Gabriela Nut, Ioana Chiorean, Petru Blaga \\ Applied Mathematics Department, Babes-Bolyai University, Cluj Napoca, Romania \\ Email:djantai@yahoo.com, ioana@math.ubbcluj.ro,blaga@math.ubbcluj.ro
}

Received March 5, 2013; revised April 17, 2013; accepted April 24, 2013

Copyright (C) 2013 Gabriela Nut et al. This is an open access article distributed under the Creative Commons Attribution License, which permits unrestricted use, distribution, and reproduction in any medium, provided the original work is properly cited.

\begin{abstract}
We use the local Fourier analysis to determine the properties of the multigrid method when used in modeling the skin penetration of a drug. The analyses of these properties can be very in designing an efficient structure of the multigrid method and in comparing the element and finite difference discretization techniques. After the theoretical results obtained, we also present some numerical results for a problem for which the solution is known.
\end{abstract}

Keywords: Time Dependent Convection Diffusion; Multigrid Method; Finite Element and Finite Differences Discretization

\section{Introduction}

In this paper we present an eoretical study of the smoothing, convergence and error reduction properties of the multigrid method for a time dependent convection diffusion equation. This is an equation that arises in the mathematical modeling of many physical phenomena, which makes the efficient numerical solution very important.

The equation studied here models the transport of molecules through the layers of the skin, until it reacheas the blood stream. The parameters used for the diffusion coefficients are smaller by several order than those of the convection, thus the equation is a convection dominated one.

The discretization of the differential equation is realized by two different methods: the finite difference method [1] with Euler backward discretization and the Galerkin finite element method [2,3].

The system obtained after the discretization process is solved using the multigrid method. This method was first introduced by Fedorenko [4,5]. The first practical results and efficiency of the method were given by Brandt $[6,7]$. The theory of multigrid convergence is well established for the Poisson equation [8-10]. In more recent articles the convergence has been studied for the convectiondiffusion equation $[11,12]$.

The novelty in this paper is that we study the smoothing factor, asimptotic convergence factor and the error reduction factor of the multigrid method for a time de- pendent convection-diffusion equation, on a domain comprising three layers with different physical properties. The analyse is performed using the local Fourier techniques $[9,13]$ which represents a good tool for constructing efficient multigrid methods for a given differential equation.

We also determined the error obtained for a given solution of the model problem, using the multigrid method on different number of grid levels, for both discretization methods mentioned above.

\section{Mathematical Model}

$$
\left\{\begin{array}{cc}
c \frac{\partial u(\boldsymbol{x}, t)}{\partial t}+\boldsymbol{v} \cdot \nabla u(\boldsymbol{x}, t) & t \geq 0, \boldsymbol{x} \in \Omega \\
=\boldsymbol{d} \Delta u(\boldsymbol{x}, t)+\alpha u(\boldsymbol{x}, t)+f(\boldsymbol{x}), & \\
u\left(\boldsymbol{x}_{0}, t\right)=u_{0}, & t \geq 0
\end{array}\right.
$$

$u(x, t)$ represents the concentration of the substance transported through the blood stream, $\boldsymbol{v}$ is the vector of convection coefficients and $\boldsymbol{d}$ is the vector of diffusion coefficients.

The substance which is transported through the skin is applied at the surface on an area with a radius of a few centimeters. The depth to which the active substance is transported by the diffusion and convection process is of the order of nanometers, thus much smaller than the radius of the surface where it is appplied. As a consequence, the problem can be reduced to the unidimen- 
sional case. From this point on, the variable $x$ will represent the depth where the concentration is to be calculated, and the vectors $\boldsymbol{v}$ and $\boldsymbol{d}$ are the coefficients in different layers of the skin.

The concentration of the substance applied on the skin is known, and the amount of it is sufficiently large to be constant at any moment of time $t$ :

$$
u(0, t)=u_{0}, t \geq 0
$$

this being the initial condition of the problem.

On the frontiers between the skin layers the law of flux conservation gives:

$$
\llbracket-d \frac{\partial u(x, t)}{\partial x} \rrbracket=0, x=x_{0 i}, i=1,2, \cdots, n_{d}, t \geq 0,
$$

$n_{d}$ is the number of layers where the diffusion takes place and:

$$
\llbracket a(x, t) \rrbracket=a\left(x^{+}, t\right)-a\left(x^{-}, t\right) .
$$

After the discretization process, the system obtained from the Equation (1) has the form:

$$
\begin{gathered}
\left\{\begin{array}{l}
q_{0} u_{i}+q_{1} u_{i-1}+q_{2} u_{i+1}=f_{i}, i=1,2, \cdots, N, \\
u_{1}=u_{0}, u_{N}=0
\end{array}\right. \\
u_{i}=u\left(x_{i}, t\right), x_{i} \in G_{h}=\{k h, k \in \mathbb{Z}, h=(b-a) /(N+1)\} .
\end{gathered}
$$

If the finite elements method [14] is used, the weak formulation of Equation (1) gives:

$$
\begin{aligned}
& \int_{0}^{b} c \frac{\mathrm{d} u}{\mathrm{~d} t} v \mathrm{~d} x+\int_{0}^{b} v \frac{\mathrm{d} u}{\mathrm{~d} x} v \mathrm{~d} x \\
& =\int_{0}^{b} d \frac{\mathrm{d}^{2} u}{\mathrm{~d} x^{2}} v \mathrm{~d} x+\int_{0}^{b} \alpha u v \mathrm{~d} x+\int_{0}^{b} f v \mathrm{~d} x,
\end{aligned}
$$

$v$ is a function that has a derivative of order 1 and is square-integrable on $\Omega=[0, b]$. The functions $u$ and $v$ are approximated using the continuous functions $\Phi_{i}: \Phi_{i}\left(x_{j}\right)=\delta_{i j}, i, j=1, \cdots, N, N$ being the number of interior points of the grid on level $l$, through the relations: $u \approx \sum_{j=1}^{N} u_{j} \Phi_{j}, v \approx \sum_{j=1}^{N} v_{i} \Phi_{i}$. Replacing the functions $u$ and $v$ with these approximates and using the standard integration-by-parts formula the equation becomes:

$$
\begin{aligned}
& \sum_{i=1}^{N} \sum_{j=1}^{N}\left(\int_{0}^{b} c \Phi_{j} \Phi_{i} \frac{\mathrm{d} u_{j}}{\mathrm{~d} t} v_{i} \mathrm{~d} x+\int_{0}^{b} v \frac{\mathrm{d} \Phi_{j}}{\mathrm{~d} x} \Phi_{i} u_{j} v_{i} \mathrm{~d} x\right. \\
& \left.+\int_{0}^{b} d \frac{\mathrm{d} \Phi_{j}}{\mathrm{~d} x} \frac{\mathrm{d} \Phi_{i}}{\mathrm{~d} x} u_{j} v_{i} \mathrm{~d} x-\int_{0}^{b} \alpha \Phi_{j} \Phi_{i} u_{j} v_{i} \mathrm{~d} x\right) \\
& =\sum_{i=1}^{N} \int_{0}^{b} f \Phi_{i} v_{i} \mathrm{~d} x
\end{aligned}
$$

or:

$$
\begin{aligned}
& \sum_{j=i-1}^{i+1}\left(c_{i j} c \frac{u_{j}-u_{j}^{\text {ant }}}{\Delta t}+v v_{i j}+\boldsymbol{d} d_{i j}-\alpha a_{i j}\right) u_{j}=f_{i}, \\
& a_{i j}=c_{i j}=\int_{0}^{b} \Phi_{j} \Phi_{i} \mathrm{~d} x ; v_{i j}=\int_{0}^{b} \frac{\mathrm{d} \Phi_{j}}{\mathrm{~d} x} \Phi_{i} \mathrm{~d} x ; \\
& d_{i j}=\int_{0}^{b} \frac{\mathrm{d} \Phi_{j}}{\mathrm{~d} x} \frac{\mathrm{d} \Phi_{i}}{\mathrm{~d} x} \mathrm{~d} x ; f_{i}=\int_{0}^{b} f \Phi_{i} \mathrm{~d} x, i, j=1, \cdots, N
\end{aligned}
$$

Computing the integrals from (4) for

$$
\Phi_{i}(x)= \begin{cases}\frac{x-x_{i-1}}{x_{i}-x_{i-1}}, & x \in\left[x_{i-1}, x_{i}\right] \\ \frac{x_{i+1}-x}{x_{i+1}-x_{i}}, & x \in\left[x_{i}, x_{i+1}\right] \\ 0, & x \notin\left[x_{i-1}, x_{i+1}\right]\end{cases}
$$

the coefficients in the system (3) are:

$$
\begin{aligned}
& q_{0}=-\frac{2 \alpha h}{3}+\frac{2 c h}{3 \mathrm{~d} t}+\frac{2 d}{h}, \\
& q_{1}=-\frac{\alpha h}{6}+\frac{c h}{6 \mathrm{~d} t}-\frac{v}{2}-\frac{d}{h}, \\
& q_{2}=-\frac{\alpha h}{6}+\frac{c h}{6 \mathrm{~d} t}+\frac{v}{2}-\frac{d}{h}, \\
& f_{i}=f\left(x_{i}\right)+\frac{c h}{6 \mathrm{~d} t}\left(u_{i-1}^{\text {ant }}+4 u_{i}^{\text {ant }}+u_{i+1}^{\mathrm{ant}}\right) .
\end{aligned}
$$

For the finite differences method using the explicit backward Euler scheme:

$$
\begin{aligned}
& c \frac{u_{i}-u_{i}^{\mathrm{ant}}}{\Delta t}+v \frac{u_{i+1}-u_{i-1}}{2 h} \\
& =d \frac{u_{i+1}-2 u_{i}+u_{i-1}}{h^{2}}+\alpha u_{i}+f\left(x_{i}\right), i=1, \cdots, N
\end{aligned}
$$

the coefficients for the system (3) will be:

$$
\begin{aligned}
& q_{0}=-\alpha+\frac{c}{\mathrm{~d} t}+\frac{2 d}{h^{2}}, q_{1}=-\frac{v}{2 h}-\frac{d}{h^{2}}, \\
& q_{2}=\frac{v}{2 h}-\frac{d}{h^{2}}, f_{i}=f\left(x_{i}\right)+c \frac{u_{i}^{\text {ant }} h}{\mathrm{~d} t} .
\end{aligned}
$$

In the nodes that are on the frontiers between different layers of the skin $\left(x_{0 i}, i=1,2, \cdots, n d\right)$, the law of flux conservation (2) becomes:

$$
\begin{aligned}
& d\left(x_{i}^{-}\right) \frac{u_{i, t}-u_{i-1, t}}{h}=d\left(x_{i}^{+}\right) \frac{u_{i+1, t}-u_{i, t}}{h}, \\
& i=1,2, \cdots, n d .
\end{aligned}
$$

In these points the system (3) has the coefficients:

$$
\begin{aligned}
& q_{0}=\frac{1}{h}\left(d\left(x_{i}^{-}\right)+d\left(x_{i}^{+}\right)\right), q_{1}=-\frac{1}{h} d\left(x_{i}^{-}\right), \\
& q_{2}=-\frac{1}{h} d\left(x_{i}^{+}\right), f_{i}=0 .
\end{aligned}
$$




\section{The Components of the Multigrid Method}

For the components of the multigrid method we give in the following the matrices associated to their operators, needed for the local Fourier analysis of the convergence. The essential property used by this method is the fact that the discretiztion of the problem leads to a system that has the eigenvectors equals to the Fourier modes and when the multigrid components have a block structure when computed in the Fourier basis, the analysis of the multigrid method is reduced to the one of diagonal blocks of small size.

\subsection{The Matrix of an Operator}

If $A$ is an operator that can be described by a difference stencil:

$$
A=\llbracket a_{1} \quad a_{2} \quad a_{3} \rrbracket
$$

meaning that:

$$
A u(x)=a_{1} u(x-h)+a_{2} u(x)+a_{3} u(x+h), x \in G_{h},
$$

then the functions $\varphi(\theta, x)=\mathrm{e}^{\frac{\mathrm{i} \theta x}{h}}, \theta \in[-\pi, \pi), x \in G_{h}$ are the eigen functions of $A$ :

$$
A \varphi(\theta, x)=\tilde{A}(\theta) \varphi(\theta, x), x \in G_{h},
$$

and:

$$
\tilde{A}(\theta)=a_{1} \mathrm{e}^{-\mathrm{i} \theta}+a_{2}+a_{3} \mathrm{e}^{\mathrm{i} \theta}
$$

are the eigenvalues of $A$.

As $\varphi(\theta, x)=\varphi(\theta+2 k \pi, x)$, it is sufficient to take $\theta \in[-\pi, \pi)$.

If $\theta \in\left[-\frac{\pi}{2}, \frac{\pi}{2}\right)=T^{\text {low }}$, the set of low frequencies, then:

$$
\bar{\theta}=\left\{\begin{array}{l}
\theta+\pi \text { if } \theta<0 \\
\theta-\pi \text { if } \theta>0
\end{array} \in T^{\text {high }},\right.
$$

$T^{\text {high }}=[-\pi, \pi)-T^{\text {low }}$ is the set of high frequencies.

Using the above notations, for an arbitrary function $\Psi(x)=\alpha \varphi(\theta, x)+\beta \varphi(\bar{\theta}, x)$, the operator $A$ applied to $\Psi$ gives:

$$
A \Psi(x)=(\varphi(\theta, x) \quad \varphi(\bar{\theta}, x)) \cdot \hat{A} \cdot\left(\begin{array}{l}
\alpha \\
\beta
\end{array}\right)
$$

and $\hat{A}$ represents the matrix associated with the operator $A$.

\subsection{The Operator of the Discretized System}

$L_{h}=\llbracket \begin{array}{lll}q_{1} & q_{0} & q_{2}\end{array} \rrbracket$ from system (3) applied to a function $\Psi$ will give:

$$
\begin{aligned}
L_{h} \Psi(x) & =\alpha \tilde{L}_{h}(\theta) \varphi(\theta, x)+\beta \tilde{L}_{h}(\bar{\theta}) \varphi(\bar{\theta}, x) \\
& =\left(\begin{array}{ll}
\varphi(\theta, x) & \varphi(\bar{\theta}, x)
\end{array}\right)\left(\begin{array}{c}
\alpha \tilde{L}_{h}(\theta) \\
\beta \tilde{L}_{h}(\bar{\theta})
\end{array}\right) \\
& =\left(\begin{array}{ll}
\varphi(\theta, x) & \varphi(\bar{\theta}, x)
\end{array}\right)\left(\begin{array}{cc}
\tilde{L}_{h}(\theta) & 0 \\
0 & \tilde{L}_{h}(\bar{\theta})
\end{array}\right)\left(\begin{array}{l}
\alpha \\
\beta
\end{array}\right),
\end{aligned}
$$

where $\tilde{L}(\theta)=q_{0}+q_{1} \mathrm{e}^{-\mathrm{i} \theta}+q_{2} \mathrm{e}^{\mathrm{i} \theta}$.

Thus the matrix of $L_{h}$ is:

$$
\hat{L}_{h}=\left(\begin{array}{cc}
\tilde{L}_{h}(\theta) & 0 \\
0 & \tilde{L}_{h}(\bar{\theta})
\end{array}\right) .
$$

\subsection{Pre- and Post- Smoother}

The Gauss-Seidel red-black method is used before and after the coarse grid correction, and reduces well the high frequency components of the error. The smoothing operator has two components of Jacobi type:

$$
\begin{aligned}
S_{h}^{\text {red }} \varphi(\theta, x) & = \begin{cases}\left(1-\frac{\omega}{q_{0}} \tilde{L}(\theta)\right) \varphi(\theta, x), x \in G^{\text {red }} \\
\varphi(\theta, x), & x \in G^{\text {black }}\end{cases} \\
& =\frac{1}{2}((a+1) \varphi(\theta, x)+(a-1) \varphi(\bar{\theta}, x)) . \\
S_{h}^{\text {black }} \varphi(\theta, x) & = \begin{cases}\varphi(\theta, x), & x \in G^{\text {red }} \\
\left(1-\frac{\omega}{q_{0}} \tilde{L}(\theta)\right) \varphi(\theta, x), x \in G^{\text {black }}\end{cases} \\
& =\frac{1}{2}((1+b) \varphi(\theta, x)+(1-b) \varphi(\bar{\theta}, x)) .
\end{aligned}
$$

In the relations above:

$$
a=1-\frac{\omega}{q_{0}} \tilde{L}(\theta), b=1-\frac{\omega}{q_{0}} \tilde{L}(\bar{\theta}) .
$$

As:

$$
\begin{aligned}
& S_{h} \Psi(\theta, x)=S_{h}^{\text {black }} S_{h}^{\text {red }} \Psi(\theta, x) \\
& =(\varphi(\theta, x) \varphi(\bar{\theta}, x)) \cdot \frac{1}{4}\left(\begin{array}{cc}
1+a & 1-b \\
1-a & b+1
\end{array}\right)\left(\begin{array}{ll}
a+1 & b-1 \\
a-1 & b+1
\end{array}\right)\left(\begin{array}{l}
\alpha \\
\beta
\end{array}\right),
\end{aligned}
$$

the matrix of the smoother will be:

$$
\begin{aligned}
& \hat{S}_{h}=\hat{S}_{h}^{\text {black }} \hat{S}_{h}^{\text {red }}= \\
& \frac{1}{4}\left(\begin{array}{cc}
(a+1)^{2}-(a-1)(b-1) & (b-1)(a-b) \\
-(a-1)(a-b) & (b+1)^{2}-(a-1)(b-1)
\end{array}\right)
\end{aligned}
$$

\subsection{Restriction of the Defect}

Full-weighting restriction is used as a fine to coarse grid transfer operator : 


$$
\begin{aligned}
& I_{h}^{2 h}=\frac{1}{2} \llbracket \begin{array}{lll}
1 & 2 & 1
\end{array} \rrbracket ; \\
& I_{h}^{2 h} \Psi(x)= \\
& =\alpha I_{h}^{2 h} \varphi_{h}(\theta, x)+\beta I_{h}^{2 h} \varphi_{h}(\bar{\theta}, x) \\
& =\alpha \tilde{I}_{h}^{2 h}(\theta) \varphi_{2 h}(2 \theta, x) \\
& +\beta \tilde{I}_{h}^{2 h}(\theta) \varphi_{2 h}(2 \bar{\theta}, x) .
\end{aligned}
$$

As

$$
\begin{aligned}
\varphi_{2 h}(2 \bar{\theta}, x) & =\mathrm{e}^{-\frac{2(\theta \pm \pi) x}{2 h}}=\mathrm{e}^{-\frac{2 \theta x}{2 h}} \\
& =\varphi_{2 h}(2 \theta, x) \text { for } x \in G_{2 h},
\end{aligned}
$$

the restriction operator applied to the function $\Psi(x)$, $x \in G_{h}$ will give:

$$
\begin{aligned}
I_{h}^{2 h} \Psi(x) & =\left(\alpha \tilde{I}_{h}^{2 h}(\theta)+\beta \tilde{I}_{h}^{2 h}(\theta)\right) \varphi_{2 h}(2 \theta, x) \\
& =\varphi_{2 h}(2 \theta, x)\left(\tilde{I}_{h}^{2 h}(\theta) \quad \tilde{I}_{h}^{2 h}(\bar{\theta})\right)\left(\begin{array}{l}
\alpha \\
\beta
\end{array}\right),
\end{aligned}
$$

with $\tilde{I}_{h}^{2 h}(\theta)=\frac{1}{2}\left(\mathrm{e}^{-\mathrm{i} \theta}+2+\mathrm{e}^{\mathrm{i} \theta}\right)$.

Thus the restriction operator has the matrix:

$$
\hat{I}_{h}^{2 h}=\frac{1}{2}(1+\cos \theta \quad 1+\cos \bar{\theta}) .
$$

\subsection{Solution on the Coarse Grid}

In the two-grid method, the exact solution on the coarse grid is required. After the restriction of the defect, the function on the grid $G_{2 h}$ has the form $\Psi_{2 h}(x)=\alpha_{2 h} \varphi_{2 h}(2 \theta, x) \quad(12,13)$. Thus:

$$
L_{2 h}^{-1} \Psi_{2 h}=\frac{\alpha_{2 h}}{\tilde{L}_{2 h}(2 \theta)} \varphi_{2 h}(2 \theta, x),
$$

wherefrom the matrix of the operator is:

$$
\hat{L}_{2 h}^{-1}(2 \theta)=\frac{1}{\tilde{L}_{2 h}(2 \theta)} .
$$

\subsection{Prolongation}

The coarse to fine interpolation operator used is the bilinear interpolation:

$$
\begin{aligned}
& I_{2 h}^{h} \varphi_{2 h}(2 \theta, x) \\
& = \begin{cases}\varphi_{2 h}(2 \theta, x), & \frac{x}{h}=2 k \in \mathbf{Z}, \\
\frac{\varphi_{2 h}(2 \theta, x+h)+\varphi_{2 h}(2 \theta, x-h)}{2}, & \frac{x}{h}=2 k+1 \in \mathbf{Z},\end{cases} \\
& =\frac{1}{2}(1+\cos \theta) \varphi_{h}(\theta, x)+\frac{1}{2}(1+\cos \bar{\theta}) \varphi(\bar{\theta}, x) .
\end{aligned}
$$

From this relation it follows that:

$$
\begin{aligned}
I_{2 h}^{h} \Psi_{2 h}(x) & =I_{2 h}^{h} \alpha_{2 h} \varphi_{2 h}(2 \theta, x) \\
& =\alpha_{2 h} \frac{1}{2}(1+\cos \theta) \varphi_{h}(\theta, x) \\
& +\alpha_{2 h} \frac{1}{2}(1+\cos \bar{\theta}) \varphi(\bar{\theta}, x)
\end{aligned}
$$

and the matrix of the prolongation operator is:

$$
\hat{I}_{2 h}^{h}=\frac{1}{2}\left(\begin{array}{c}
1+\cos \theta \\
1+\cos \bar{\theta}
\end{array}\right) .
$$

\subsection{Two-Grid Operator}

The multigrid method $[8,15]$ is a combination between a relaxation method (that reduces very well the high frequency components of the error, but is slowly convergent because of the low frequency components) and the coarse grid correction (which has complementary properties to the smoother).

The matrices from (9), (11), (14), (15) and (16) are used to create the two-grid operator for the multigrid method:

where:

$$
\hat{M}_{h}^{2 h}=\hat{S}_{h}^{v_{2}} \hat{K}_{h}^{2 h} \hat{S}_{h}^{\nu_{1}}
$$

$$
\hat{K}_{h}^{2 h}=\hat{I}_{h}-\hat{I}_{2 h}^{h}\left(\hat{L}_{2 h}\right)^{-1} \hat{I}_{h}^{2 h} \hat{L}_{h}
$$

is the matrix of the coarse grid correction operator.

It has been proven [9] that it is sufficient to derive the convergence properties for the two-grid method and the multigrid method will have similar properties. As a consequence, the following factors are defined for the two-grid operator.

\section{Asimptotic convergence factor}

$$
\begin{aligned}
& \rho_{\text {loc }}\left(M_{h}^{2 h}\right) \\
& =\sup \left\{\rho_{\text {loc }}\left(\hat{M}_{h}^{2 h}(\theta)\right), \theta \in T^{\text {low }}=\left[-\frac{\pi}{2}, \frac{\pi}{2}\right], \theta \notin \Lambda\right\} .
\end{aligned}
$$

\section{Error reduction factor}

$$
\begin{aligned}
& \sigma_{\text {loc }}\left(M_{h}^{2 h}\right) \\
& =\sup \left\{\left\|\left(\hat{M}_{h}^{2 h}(\theta)\right)\right\|, \theta \in T^{\text {low }}=\left[-\frac{\pi}{2}, \frac{\pi}{2}\right], \theta \notin \Lambda\right\} .
\end{aligned}
$$

Here: $\|\cdot\|$ denotes the spectral norm associated with the Euclidian vector norm in $\mathbb{C}^{2}$, and

$$
\Lambda=\left\{\theta \in\left[-\frac{\pi}{2}, \frac{\pi}{2}\right], \tilde{L}_{h}(\theta)=0 \text { or } \tilde{L}_{2 h}(\theta)=0\right\} .
$$

\section{Smoothing factor}

$$
\begin{gathered}
\mu_{\text {loc }}\left(S_{h}, v\right)=\sup \left\{\sqrt[v]{\rho_{\text {loc }}\left(\hat{S}_{h}^{v_{2}}(\theta) \hat{Q}_{h}^{2 h} \hat{S}_{h}^{v_{1}}(\theta)\right)},\right. \\
\left.\theta \in T^{\text {low }}=\left[-\frac{\pi}{2}, \frac{\pi}{2}\right]\right\} .
\end{gathered}
$$


where $\rho_{\text {loc }}(\hat{S}(\theta))$ is the spectral radius of the matrix $\hat{S}(\theta), v=v_{1}+v_{2}$.

$Q_{h}^{2 h}$, introduced in [9], is an "ideal" coarse grid operator that anihilates the low frequency error components and leaves the high frequency components unchanged:

$$
Q_{h}^{2 h} \varphi(\theta, \cdot)=\left\{\begin{array}{l}
0, \quad \text { if } \theta \in T^{\text {low }}=\left[-\frac{\pi}{2}, \frac{\pi}{2}\right] \\
\varphi(\theta, \cdot), \text { if } \theta=\bar{\theta} \in T^{\text {high }}
\end{array}\right.
$$

and:

$$
\hat{Q}_{h}^{2 h}(\theta)=\left(\begin{array}{ll}
0 & 0 \\
0 & 1
\end{array}\right), \theta \in T^{\text {low }} .
$$

Since $\rho_{\text {loc }}\left(\hat{S}_{h}^{v_{2}}(\theta) \hat{Q}_{h}^{2 h} \hat{S}_{h}^{v_{1}}(\theta)\right)=\rho_{\text {loc }}\left(\hat{Q}_{h}^{2 h} \hat{S}_{h}^{v}(\theta)\right)$,

$$
\mu_{\text {loc }}\left(S_{h}, v\right)=\sup \left\{\sqrt[v]{\rho_{\text {loc }}\left(\hat{Q}_{h}^{2 h} \hat{S}_{h}^{v}(\theta)\right)}, \theta \in T^{\text {low }}\right\} .
$$

\section{Local Fourier Analysis Results for the Studied Problem}

\subsection{Smoothing Factor}

If $\omega=1$ then the matrix (11) of the smoother after $v=v_{1}+v_{2}$ steps is:

$$
\hat{S}_{h}^{v}(\theta)=\frac{1}{4^{v-1}}(a-b)^{2(v-1)} \hat{S}_{h}(\theta)
$$

and has the eigenvalues:

$$
\lambda_{1}=0, \lambda_{2}=\frac{(a-b)^{2(v-1)}}{2^{2 v}}\left[(b+1)^{2}-(a-1)(b-1)\right],
$$

$a$ and $b$ being given in (10),

$$
\begin{aligned}
& \left|\lambda_{2}\right|^{2}=\frac{\left[\left(q_{1}-q_{2}\right)^{2}+4 q_{1} q_{2} \cos ^{2} \theta\right]^{2 v-1}}{4 q_{0}^{4 v}} \\
& \frac{\left[q_{0}^{2}+\left(q_{1}-q_{2}\right)^{2}+4 q_{1} q_{2} \cos ^{2} \theta+2 q_{0}\left(q_{1}+q_{2}\right) \cos \theta\right]}{4 q_{0}^{4 v}}
\end{aligned}
$$

For $\theta \in T^{\text {low }}$, the eigen value $\lambda_{2}$ attains its maximum absolute value:

$$
\left|\lambda_{2}\right|=\left|\frac{q_{0}+q_{1}+q_{2}}{2 q_{0}}\left(\frac{q_{1}+q_{2}}{q_{0}}\right)^{2 v-1}\right|
$$

for $\theta=0$. The smoothing factor for the problem (1) is:

$$
\mu_{\text {loc }}\left(S_{h}, v\right)=\left(\frac{q_{1}+q_{2}}{q_{0}}\right)^{2} \sqrt[v]{\frac{q_{0}+q_{1}+q_{2}}{2\left(q_{1}+q_{2}\right)}} .
$$

\begin{tabular}{|c|c|c|c|c|c|}
\hline \multicolumn{6}{|c|}{ Finite differences } \\
\hline & $v=1$ & $v=2$ & $v=3$ & $v=4$ & $v=5$ \\
\hline$l=3$ & 0.0058 & $8.8272 \times 10^{-4}$ & $4.7260 \times 10^{-4}$ & $3.4581 \times 10^{-3}$ & $2.8670 \times 10^{-3}$ \\
\hline$l=4$ & 0.0215 & 0.0066 & 0.0044 & 0.0037 & 0.0032 \\
\hline$l=5$ & 0.0667 & 0.0409 & 0.0348 & 0.0321 & 0.0306 \\
\hline$l=6$ & 0.1225 & 0.1504 & 0.1611 & 0.1667 & 0.1701 \\
\hline$l=7$ & 0.1250 & 0.2296 & 0.3098 & 0.3598 & 0.3937 \\
\hline \multicolumn{6}{|c|}{ Finite element method } \\
\hline & $v=1$ & $v=2$ & $v=3$ & $v=4$ & $v=5$ \\
\hline$l=3$ & 0.3493 & 0.2801 & 0.2603 & 0.2509 & 0.2454 \\
\hline$l=4$ & 0.2809 & 0.2125 & 0.1937 & 0.1849 & 0.1798 \\
\hline$l=5$ & 0.0991 & 0.0533 & 0.0434 & 0.0391 & 0.0368 \\
\hline$l=6$ & 0.1042 & 0.0955 & 0.0928 & 0.0915 & 0.0907 \\
\hline$l=7$ & 0.1250 & 0.2291 & 0.3031 & 0.3486 & 0.3792 \\
\hline
\end{tabular}

Here, $q_{0}, q_{1}, q_{2}$ are the coefficients given in (5)-(7). For $a=10^{-4}, c t=1, d_{1}=1 \times 10^{-12}, d_{2}=1 \times 10^{-10}, d_{3}=$ $3 \times 10^{-10}, v_{1}=1 \times 10^{-9}, v_{2}=1 \times 10^{-6}, v_{3}=1 \times 10^{-6}$, the smoothing factors for the Gauss-Seidel relaxation method are presented in Table 1.

The data from Table 1 show that:

Table 1. The smoothig factor as a function of $v=v_{1}+v_{2}$ and $l$. 
- the Gauss-Seidel red-black relaxation method is a very good smoother for this problem as the smoothing factors in the cases presented here are $\leq 0.5$;

- both the discretization methods lead to good smoothing factors. The finite element method seems slightly more appropriate when the number of grids used in the multigrid method is bigger;

- the number of relaxation steps before and after the coarse grid correction should not be too big as the smoothing factor increases with $v$.

\subsection{Asimptotic Convergence Factor and Error Reduction Factor}

For the multigrid method having the components described in (9-16), the matrix of the two-grid operator for the problem (1) is:

$$
\begin{aligned}
& \hat{M}_{h}^{2 h}=\hat{S}_{h}^{v_{2}}(\theta)\left[\left(\begin{array}{ll}
1 & 0 \\
0 & 1
\end{array}\right)-\frac{1}{4 \tilde{L}_{2 h}(2 \theta)}\right. \\
& \left.\cdot\left(\begin{array}{ll}
(1+\cos \theta)^{2} \tilde{L}_{h}(\theta) & (1-\cos \theta)^{2} \tilde{L}_{h}(\bar{\theta}) \\
(1-\cos \theta)^{2} \tilde{L}_{h}(\theta) & (1+\cos \theta)^{2} \tilde{L}_{h}(\bar{\theta})
\end{array}\right)\right] \hat{S}_{h}^{v_{1}}(\theta)
\end{aligned}
$$

For $\theta \in\left[-\frac{\pi}{2}, \frac{\pi}{2}\right]$ the corresponding asimptotic convergence factor and error reduction factor have been computed from the matrix (26) and are given in Table 2 for different numbers of pre- and post- smoothing steps.

The data from Table 2 show that the multigrid method is very rapidly convergent: if at least one smoothing step is performed before and after the coarse grid correction, then the error is reduced by at least a $10^{-3}$ factor per multigrid cycle.

\section{Numerical Results}

The problem (1) has been solved on a domain containing tree layers with different diffusion and convection coefficients $([16,17])$.

The error was computed for the exact solution

$$
\begin{aligned}
& u_{e x}(x, t)=x^{2}+t, \max (\mid \text { ue }(x, t) \mid)=1.44 \times 10^{3}, \\
& x \in \Omega=[0,1620 \mathrm{~nm}], t \in[0,24 \mathrm{~min}]
\end{aligned}
$$

The time step in the discretization process has been $\mathrm{d} t=60 \mathrm{~s}$. Figure 1, Figure 2 and the Table 3 represent

\begin{tabular}{|c|c|c|c|c|}
\hline \multirow{2}{*}{$\begin{array}{l}\text { Number of } \\
\text { smoothig steps }\end{array}$} & \multicolumn{2}{|c|}{ Finite differences } & \multicolumn{2}{|c|}{ Finite element method } \\
\hline & $\rho_{\text {loc }}\left(M_{h}^{2 h}\right)$ & $\sigma_{\text {loc }}\left(M_{h}^{2 h}\right)$ & $\rho_{\text {loc }}\left(M_{h}^{2 h}\right)$ & $\sigma_{\text {loc }}\left(M_{h}^{2 h}\right)$ \\
\hline$v_{1}=0, v_{2}=1$ & 0.1224 & 0.1731 & 0.0153 & 0.1989 \\
\hline$v_{1}=1, v_{2}=0$ & 0.1224 & 0.3297 & 0.0153 & 0.2420 \\
\hline$v_{1}=1, v_{2}=1$ & 0.0225 & 0.0570 & $9.1275 \times 10^{-4}$ & 0.0037 \\
\hline$v_{1}=2, v_{2}=1$ & 0.0041 & 0.0105 & $6.8022 \times 10^{-5}$ & $2.5397 \times 10^{-4}$ \\
\hline$v_{1}=2, v_{2}=2$ & $7.5569 \times 10^{-4}$ & 0.0019 & $5.6099 \times 10^{-6}$ & $2.0141 \times 10^{-5}$ \\
\hline$v_{1}=3, v_{2}=2$ & $1.3862 \times 10^{-4}$ & $3.5209 \times 10^{-4}$ & $4.8333 \times 10^{-7}$ & $1.7152 \times 10^{-6}$ \\
\hline
\end{tabular}
the error after eight multigrid cycles, with two smoothing steps before and two after the coarse grid correction.

Table 2. Asimptotic convergence factor and error reduction factor $(I=6)$.

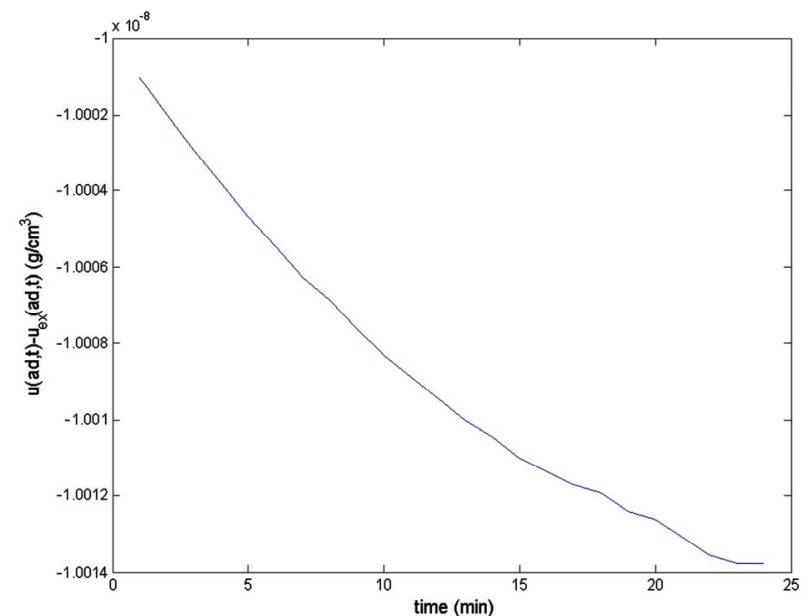

(a)

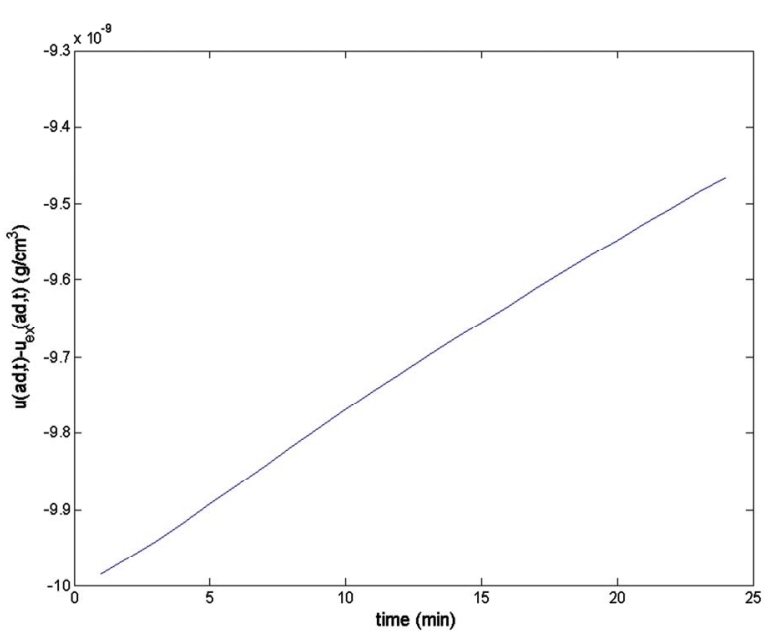

(b)

Figure 1. The multigrid error at ad $=100 \mathrm{~nm}$ in the skin for $v_{1}=1.0 \times 10^{-10}, v_{2}=1.0 \times 10^{-7}, v_{3}=1.0 \times 10^{-7}, d_{1}=1 \times 10^{-12}, d_{2}=1$ $\times 10^{-10}, d_{3}=3 \times 10^{-10} ; c=10^{4} ; a=0$. 


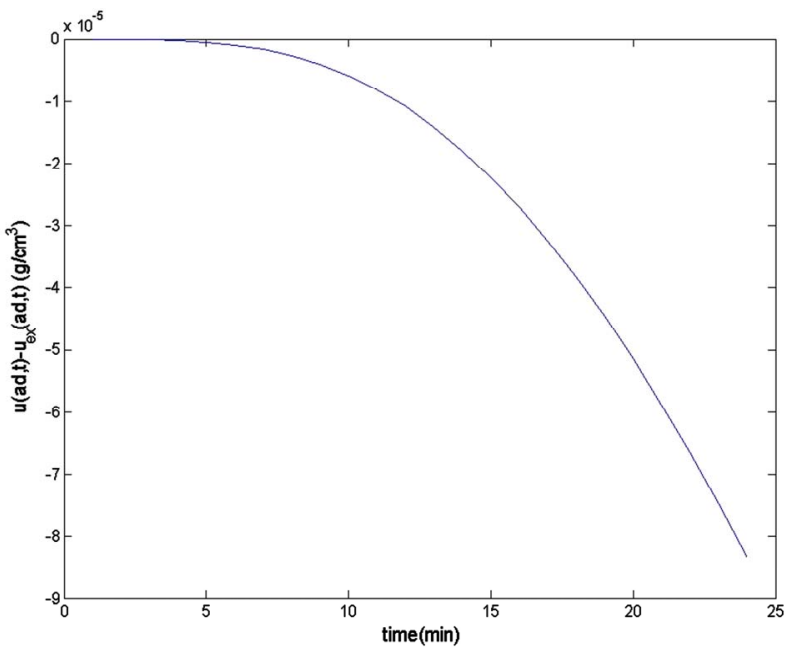

(a)

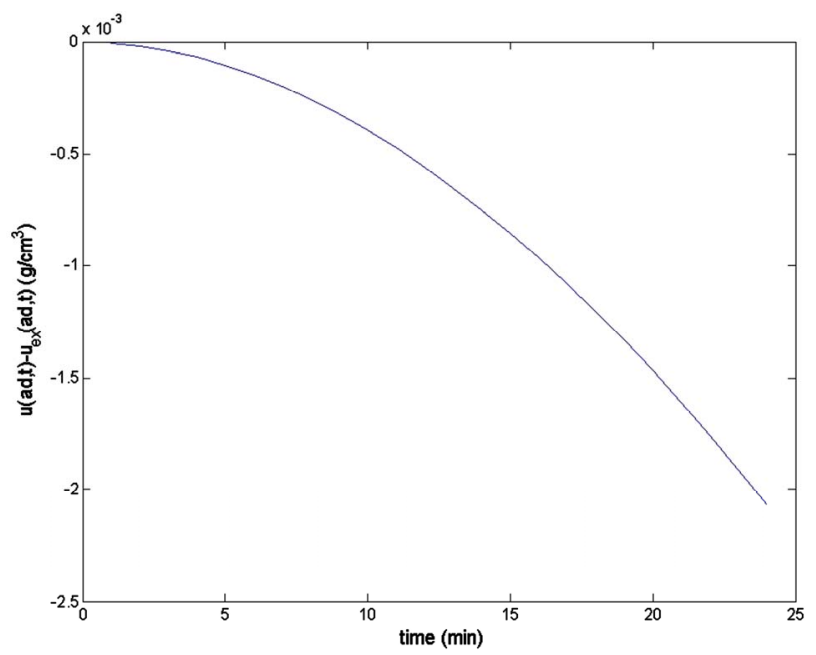

(b)

Figure 2. The multigrid error at ad $=100 \mathrm{~nm}$ in the skin for $v_{1}=1.0 \times 10^{-9}, v_{2}=1.0 \times 10^{-6}, v_{3}=1.0 \times 10^{-6}, d_{1}=1 \times 10^{-12}, d_{2}=1$ $\times 10^{-10}, d_{3}=3 \times 10^{-10} ; c=10^{3} ; a=0$.

Table 3. Multigrid error for FD and FEM.

\begin{tabular}{ccc}
\hline & \multicolumn{2}{c}{$\mathrm{FD}$} \\
\hline$=3$ & $\max _{i}\left|u\left(x_{i}\right)-u_{e x}\left(x_{i}\right)\right|$ & $\left\|u-u_{e x}\right\|$ \\
$l=4$ & $1.0000 \times 10^{-8}$ & $4.8970 \times 10^{-8}$ \\
$l=5$ & $1.0057 \times 10^{-8}$ & $3.1812 \times 10^{-8}$ \\
$l=6$ & $1.0617 \times 10^{-7}$ & $1.6775 \times 10^{-7}$ \\
$l=7$ & $8.3356 \times 10^{-7}$ & $1.9383 \times 10^{-6}$ \\
& $5.5076 \times 10^{-6}$ & $1.4308 \times 10^{-5}$ \\
$l=3$ & $\mathrm{FEM}^{-5}$ & \\
$l=4$ & $\max _{i}\left|u\left(x_{i}\right)-u_{e x}\left(x_{i}\right)\right|$ & $\left\|u-u_{e x}\right\|$ \\
$l=5$ & $9.9847 \times 10^{-9}$ & $4.7554 \times 10^{-8}$ \\
$l=6$ & $1.6648 \times 10^{-8}$ & $5.9871 \times 10^{-8}$ \\
$l=7$ & 0.1079 & 0.2457 \\
\hline
\end{tabular}

Table 3 shows the maximum absolute value of the error and the norm of the error vector corresponding to Figures 1(a) and (b), for the finite differences discretization method (FD) and finite element method (FEM).

\section{Conclusion}

We have presented a convergence and error analysis for the multigrid method applied to a time dependent diffusion-convection problem that is convection dominated. The mathematical model is applied to the study of the concentration of a solute that is transported by the blood, or the penetration of a substance through the skin layers.
The convergence analysis showed that the discretization process is better realized by the finite element method than the finite differences. Also the red-black GaussSeidel is a good smoother for the problem presented here, and needs not to be applied more than two or three times in the construction of the multigrid method.The numerical results in the previous paragraph confirmed the good convergence and error reduction as predicted by the coefficients computed with the local Fourier analysis.

\section{REFERENCES}

[1] K. W. Morton and D. F. Mayers, "Numerical Solution of Partial Differential Equations, An Introduction," Cambridge University Press, Cambridge, 2005. doi:10.1017/CBO9780511812248

[2] E. Becker, G. Carey and J. Oden, "Finite Elements. An Introduction," Prentice-Hall, Englewood Cliffs, 1981.

[3] H. C. Elman, D. J. Silvester and A. J. Wathen, "Finite Elements and Fast Iterative Solvers," Oxford University Press, Oxford, 2005.

[4] R. P. Fedorenko, "A Relaxation Method for Solving Elliptic Difference Equations," USSR Computational Mathematics and Mathematical Physics, Vol. 1, 1962, pp. 10921096.

[5] R. P. Fedorenko, "The Speed of Convergence of One Iterative Process," USSR Computational Mathematics and Mathematical Physics, Vol. 4, 1964, pp. 227-235.

[6] A. Brandt, "Multilevel Adaptive Solutions to Boundary Value Problems," Mathematics of Computation, Vol. 31, 1977, pp. 333-390.

doi:10.1090/S0025-5718-1977-0431719-X

[7] A. Brandt, "Multigrid Techniques: 1984 Guide with Applications to Fluid Dynamics," GMD-Studien Nr. 85, Gesellschaft für Matematik und Datenverarbeitung, St. Augustin, Bonn, 1984. 
[8] W. Hackbush, "Elliptic Differential Equations," SpringerVerlag, New York, 1992.

doi:10.1007/978-3-642-11490-8

[9] U. Trottenberg, C. Oosterlee and A. Schuller, "Multigrid," Elsevier Academic Press, London, 2001.

[10] P. Weseling, "An Introduction to Multigrid Method," John Wiley \& Sons, New York, 1991.

[11] M. A. Olshanski and A. Reusken, "On a Robust Multigrid Method for Connection-Diffusion Finite Element Problems."

http://www.math.uh.edu/ molshan/ftp/pub/proceed_cd.pdf

[12] A. Reusken, "Convergence Analysis of a Multigrid Method for Convection-Diffusion Equations," Numerische Mathematik, Vol. 91, No. 2, 2002, pp. 323-349. doi: $10.1007 / \mathrm{s} 002110100312$

[13] R. Wienands and W. Joppich, "Practical Fourier Analysis for Multigrid Methods," Chapman\& Hall/CRC Press, Bo- ca Raton, 2005.

[14] R. W. Lewis, P. Nithiarasu and K. N. Seetharamu, "Fundamentals of the Finite Element Method for Heat and Fluid Flow," John Wiley \& Sons Ltd, The Atrium, 2004. doi:10.1002/0470014164

[15] W. L. Briggs, V. E. Henson and S. McCormick, "A Multigrid Tutorial,"2nd Edition, Siam, Philadelphia, 2000. doi:10.1137/1.9780898719505

[16] D. Neumann, "Modeling Transdermal Absorption," Biotechnology: Pharmaceutical Aspects, Springer, New York, 2008.

[17] B. Al-Qallaf, D. Bhusan Das, D. Mori and Z. Cui, "Modelling Transdermal Delivery of High Molecular Weight Drugs from Microneedle Systems," Philosophical Transactions of the Royal Society A, Vol. 365, 2007, pp. 29512967. doi: $10.1098 /$ rsta.2007.0003 